\title{
Incorporation of genetic marker information in beef cattle breeding programs
}

\author{
F.M. Rezende \\ Instituto de Genética e Bioquímica, Universidade Federal de Uberlândia, \\ Patos de Minas, MG, Brasil
}

2012. Programa de Pós-Graduação em Qualidade e Produtividade Animal, Faculdade de Zootecnia e Engenharia de Alimentos, Universidade de São Paulo, Pirassununga, SP, Brasil. PhD thesis. Orienting Prof.: Dr. Flávio Vieira Meirelles.

DOI http://dx.doi.org/10.4238/2013.October.30.1

Corresponding author: F.M. Rezende

E-mail: frezende@ingeb.ufu.br

\begin{abstract}
The availability of molecular marker information has turned out to be an opportunity to improve animal breeding programs, by the inclusion of those effects in the estimation of breeding values. Along this perspective, the aims of present research were to compare genetic evaluation models that assume or not marker effects in the estimation of breeding values, as well as to estimate the allelic substitution effects of SNP markers, by applying six different methods (Bayesian multiple regression, Bayesian ridge regression, Bayes A, Bayes B, Bayes C $\pi$, and Bayesian Lasso), and to evaluate the impact of these effects on the reliability of breeding values and the divergences in animal classification based on classical breeding values and markerassisted breeding values. Data of 83,404 animals belonging to a Nellore beef cattle (Bos indicus) selection program, measured for post-weaning gain, scrotal circumference and muscle score, and corresponding to 116,562 animals in the relationship matrix, were used. From these animals, a set of 3160 animals with phenotypic and genealogy data available were genotyped with a panel of 106 SNP markers. Model comparison results did not clearly demonstrate the advantage of
\end{abstract}


assuming polygenic and marker effects together in genetic evaluation models; however, models that considered only the effects of markers showed the worst global fit and predictive ability. Differences observed in estimates of marker effects were due to the shrinkage process applied by each method. The incorporation of marker information in genetic evaluations, in general, increased the reliability of breeding values, mainly for the young replacement animals. Comparing the $20 \%$ best animals classified by classical breeding value and marker-assisted breeding value, the highest divergences were observed for sires and young bulls that were genotyped. In summary, although this research showed that the inclusion of very low-density SNP chip information was not able to improve the predictive ability of genetic evaluation models, it increased the reliability of breeding value estimates.

Key words: Marker-assisted selection; Bayesian inference; Molecular breeding value 